\title{
Super Resolution of Remote Sensing Images using Edge-Directed Radial Basis Functions
}

\author{
Manohar Mareboyana ${ }^{1}$, Jacqueline Le Moigne ${ }^{2}$ \\ 1. ASRC Federal, NASA Goddard, also at Bowie State University, Bowie, MD, 20715 \\ 2. $\quad$ NASA Goddard, Code 580, Greenbelt, MD 20771
}

\begin{abstract}
Edge-Directed Radial Basis Functions (EDRBF) are used to compute super resolution(SR) image from a given set of low resolution (LR) images differing in subpixel shifts. The algorithm is tested on remote sensing images and compared for accuracy with other well-known algorithms such as Iterative Back Projection (IBP), Maximum Likelihood (ML) algorithm, interpolation of scattered points using Nearest Neighbor (NN) and Inversed Distance Weighted (IDW) interpolation, and Radial Basis Functin(RBF) . The accuracy of SR depends on various factors besides the algorithm (i) number of subpixel shifted LR images (ii) accuracy with which the LR shifts are estimated by registration algorithms (iii) and the targeted spatial resolution of SR. In our studies, the accuracy of EDRBF is compared with other algorithms keeping these factors constant. The algorithm has two steps: i) registration of low resolution images and (ii) estimating the pixels in High Resolution (HR) grid using EDRBF. Experiments are conducted by simulating LR images from a input HR image with different sub-pixel shifts. The reconstructed SR image is compared with input HR image to measure the accuracy of the algorithm using sum of squared errors (SSE). The algorithm has outperformed all of the algorithms mentioned above. The algorithm is robust and is not overly sensitive to the registration inaccuracies.
\end{abstract}

Keywords - Super Resolution, Radial Basis Functions, Edge-Directed Radial Basis Functions

\section{INTRODUCTION}

Spatial resolution of remotely sensed images plays an important role in analysis and visual interpretations. Unfortunately the spatial resolution of remote sensing system cannot be increased without limit. The limiting factors are diffraction limits, noise, and spacecraft orbits. An excellent description of limiting factors of ground resolution by optical systems onboard satellites is given in [1].

Super resolution (SR) is the process of artificially increasing the spatial resolution of an image corresponding to a scene given a set of observed low resolution (LR) images of the same scene that differ in sub-pixel translation. The low resolution images of the scene provide complementary information that can be used to estimate pixel values in denser grid. Constructing a SR image from a set of LR images is basically a constrained ill-posed problem, because there is no unique solution. The interest in this area of research is spurred by the seminal paper by Yang and Huang [2] that provides an excellent review of the techniques used. They can be divided broadly into a) Frequency Domain b) Spatial Domain approaches using interpolation and statistical methods c) wavelet based, and set theoretic methods. Although frequency domain approaches are fast, they lack ability to incorporate priors in computing SR images. Wavelet techniques have been applied to super resolution following similar over all approach as frequency domain [3]. A variety of techniques exist in spatial domain approaches among which interpolation and regularization, statistical methods, and projection onto convex sets are used extensively in super resolution. The advantages of spatial domain approaches are that unconstrained motion between frames can be handled easily and a-priori knowledge can be incorporated in regularization.

A number of iterative methods such as Projection on Convex Sets (POCS) [4], Iterative Back Projection(IBP) [5] have been successfully applied to super resolution. The iterative methods are time consuming and become inefficient for large images. Statistical approaches of Maximum Likelihood Estimation (MLE) and Maximum a Posterior (MAP) [6,2] have been applied to super resolution. These methods depend on the models assumed and do not take geometrical relations betwee the motion parameters explicitly. In the following sections, we describe interpolation based techniques in 
reconstructing super resolution. In particular, interpolation using Radial Basis Functions (RBF), and Edge Directed Radial Basis Functions (EDRBF) are described. The results obtained using EDRBF on remotely sensed images are presented and compared with other popular techniques.

\section{INTERPOLATION BASED TECHNIQUES}

Consider estimating SR image, $X$ from multiple LR images $\mathrm{y}_{1}, \mathrm{y}_{2}, . . \mathrm{y}_{\mathrm{k}}$

$$
\mathrm{y}_{\mathrm{k}}=\mathrm{D} \mathrm{F}_{\mathrm{k}} \mathrm{B} \mathrm{X}+\mathrm{N}
$$

Where $\mathrm{X}$ and $\mathrm{y}_{\mathrm{k}}$ are SR and LR images, B is space-invariant blurring kernel, $\mathrm{F}_{\mathrm{k}}$ is motion operator, D is down sampling operator, and $\mathrm{N}$ is noise term . Combining all three operators into a single operator $\mathrm{H}_{\mathrm{k}}$ for kth frame, the above relation can be given as follows.

$$
\mathrm{y}_{\mathrm{k}}=\mathrm{H}_{\mathrm{k}} \mathrm{X}+\mathrm{N}
$$

Where $\mathrm{H}=\left[\mathrm{H}_{1}, \mathrm{H}_{2}, . . \mathrm{H}_{\mathrm{k}}\right]^{\mathrm{t}}$ and incorporates, blurring, motion and down sampling of SR image X. Considering blurring and down sampling are space-invariant, $\mathrm{X}$ can be estimated from motion operator, given $\mathrm{y}_{\mathrm{k}}$. The noise component makes the solution to this inverse problem even more difficult. Thus the interpolation techniques rely on motion operator and regularization of interpolated images to account for noise.

\subsection{Nearest Neighbor}

The simplest interpolation used in constructing a HR image from a set of LR images is to assign the closest LR image pixel value to every point in HR grid. Obviously, it is the fastest interpolation and uses a single LR-image for a given HR grid point [7].

\subsection{Interpolation from Scattered Points}

Image interpolation on uniformly gridded data is well known and a number of techniques linear, cubic, and spline interpolations [7-8] exist in literature. In the context of super resolution, the interpolation of scatter of points (nonuniformly gridded points) is applicable.

Interpolation using Inverse Distance Square (IDS) [8] are well-known.

\subsection{Interpolation using Radial Basis Function}

A radial basis function $(\mathrm{RBF})$ is a real valued function whose value depends on the distance from a given point $\mathrm{x}_{\mathrm{i}}(\mathrm{x}$ a variable not to be confused with image variable used for SR image).

$$
\emptyset\left(x, x_{i}\right)=\emptyset\left(\left\|x-x_{i}\right\|\right)
$$

When the distance function is Gaussian, the RBF is called Gaussian RBF.

$$
\emptyset\left(x, x_{i}\right)=e^{-\left(x-x_{i}\right)^{2}}
$$

The reference LR image is used to fill up every other HR grid pixels along the row and column. The remaining LR images are used to estimate the 3 missing cells of $2 \times 2$ HR grid as follows

$$
x(i)=\sum_{k=1}^{4} \emptyset(i-T(k)) * y_{k}
$$

where $\mathrm{i}$ is position of missing cell from HR grid. RBF based interpolation is used to reconstruct super resolution image in [9]. 


\section{EDGE-DIRECTED RBF INTERPOLATION}

RBF interpolation is solely based on distance of the scattered points from the point where the value is interpolated. All the points equidistant from the point weighted equally to the value. In image interpolation, the pixels along the gradient are not considered, therefore should be weighted least compared to the ones which are perpendicular to the gradient direction or along the edges. The RBF that takes into account the edge direction in the interpolation is called Edge-Directed $\mathrm{RBF}$ (EDRBF). EDRBF based interpolation is used in super-resolution from a single LR image in [10-12]. The following equation modifies the interpolation that takes the edge direction into account.

$$
x(i)=\sum_{k=1}^{4} \varnothing(i-T(k)) * \sin \left(\theta_{g}-\theta_{d}\right) * y_{k}
$$

where $\theta_{g}$ is the direction of gradient at position $i$, and $\theta_{d}$

is the direction of point, from $x(i)$.

The above equationcan be represented by a linear mapping of the LR images, $y_{k}$ into SR image, $x(i)$

$$
x=W y
$$

where $y$ is a set of $\mathrm{k}$ LR images, and $x$ is a SR image constructed using the weight matrix $W$. For resolution enhancement factor, $r$ the number of LR images $k$ with independent subpixel shifts should be equalt $r^{2}$ for optimal performance.

The SR image, $x$ is populated such that every $\mathrm{r}^{\text {th }}$ pixel along the row and column is filled by reference LR image (say, $\left.y_{1}\right)$. Now they are $\left(\mathrm{r}^{2}-1\right)$ pixels in every rxr subregion to be interpolated using. In Equation (3.2) $x$ is $\mathrm{r}^{*} \mathrm{r}$ subregion of SR image, $x$ and $\mathrm{y}$ is a vector corresponding to $\mathrm{k}$ pixels of LR images as shown in Fig. 1.

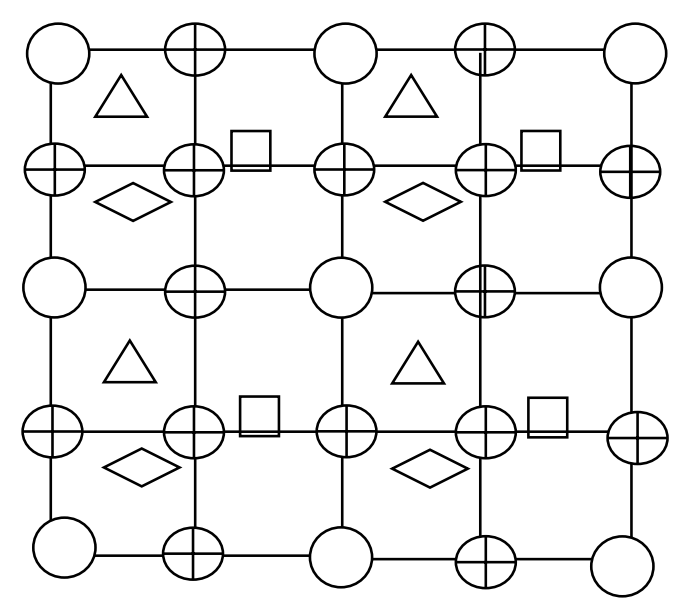

Figure 1: The LR images super imposed on HR grid:

LR Reference on HR grid

$\triangle \square \diamond$ LR Images with subpixel shifts

$\bigoplus \quad$ Interpolated image on HR grid 


\section{EXPERIMENTAL RESULTS}

Using Equation 2.2, we have simulated LR images from a single band Landsat image (pixel intensities from 5 to 155) shown in Figure 2. The LR images differ from each other in subpixel translations. The resolution enhancement factor of 2 is used in the experiments to construct SR image from LR images using different algorithms.

In the implementation of EDRBF algoritm, each element of $3 \times 4$ matrix W in Equation (3.2) denoted by $w_{\mathrm{ij}}$ is computed as follows.

$$
w_{i j}=e^{-g m(\|i-j\|} * \sin \left(\theta_{g}-\theta_{d}\right)
$$

In the above equation, $i$ is the point in the $2 \times 2$ region of SR image and $j$ is the location of subpixel shift of the LR image $j$. The gradient magnitude and direction of reference LR image is extrapolated to SR image locations and wij is computed as in Equation (4.1) where the gradient value in reference LR image is greater than a threshold (approx. 70\% maximum gradient). The parameter gm in the equation is selected for optimal results $(\mathrm{gm}=4.0)$. SR image reconstructed using EDRBF is shown in Figure 3. The error image is shown in Figure 4. The Table 1 presents the performance comparison of EDRBF with other SR algorithms implemented. Since algorithms based on MLE and IBP are far from the remaining three in terms of reconstruction accuracy, we have conducted further experiments using the other competing algorithms. In table 2, we have presented MSE in reconstruction at different subpixel shifts. With ideal shifts the SR can be reconstructed from LR image with zero MSE. In Table 2 we present MSE with given percent deviation from ideal shift and jitter (specified by variance of Gaussian random noise). As can be seen the EDRBF and RBF outperformed other algorithms with EDRBF having slightly better accuracy.

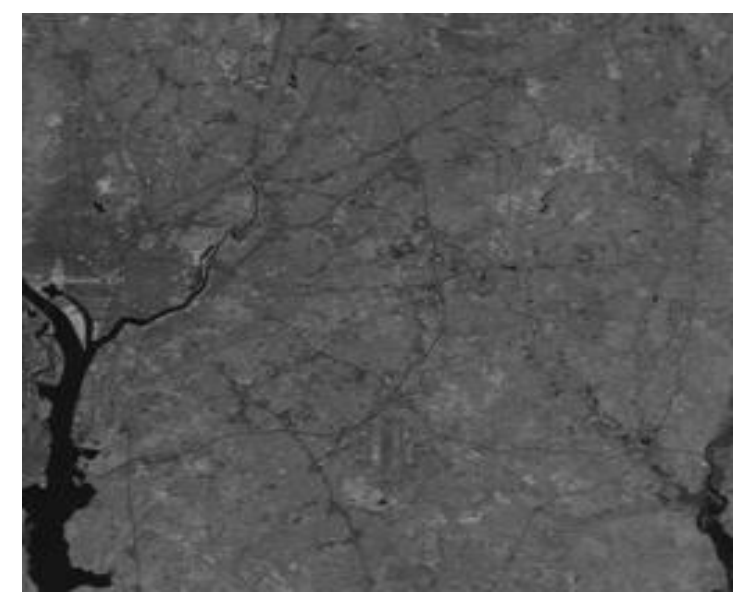

Figure 2: HR input test image

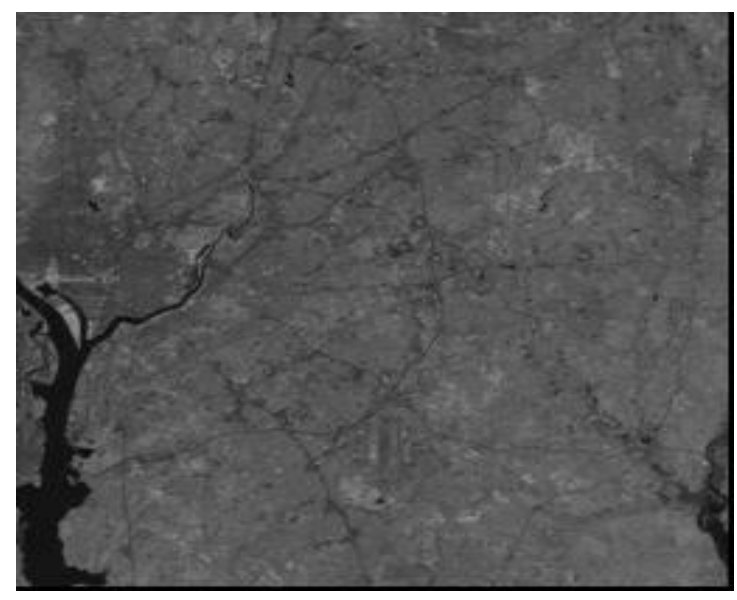

Figure 3: Reconstructed image using EDRBF 


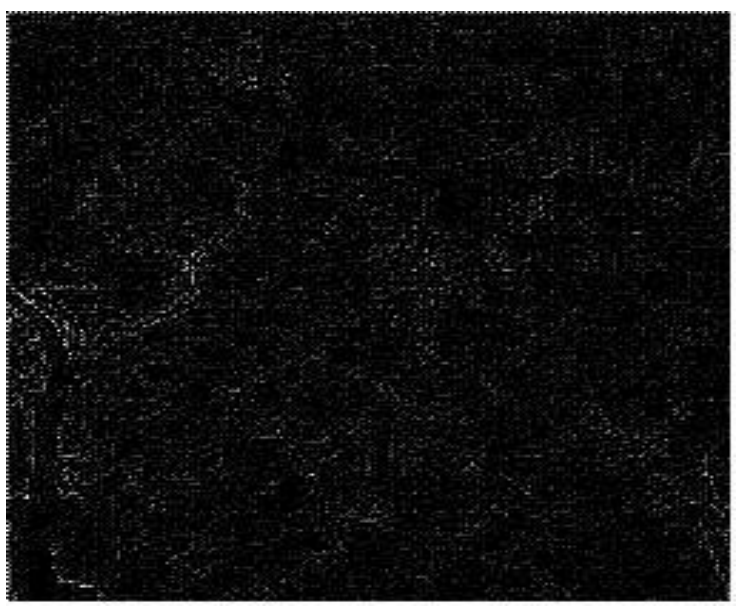

Figure 4: 20 Times Absolute Error in SR

TABLE 1

$($ Deviation from Ideal shift, Dev $=10 \%$, jitter $=0)$

\begin{tabular}{|l|l|}
\hline Algorithm & MSE \\
\hline NN(Nearest Neighbor) interpolation & 0.7453 \\
\hline IDW (inverse Distance Weighted) & 1.4266 \\
\hline MLE (Maximum Likelihood) & 2.9293 \\
\hline IBP (Iterative Back Projection) & 3.9748 \\
\hline RBF (Radial Basis Function) & 0.6926 \\
\hline EDRBF (Edge-Directed RBF) & 0.6924 \\
\hline
\end{tabular}

\section{TABLE 2}

\begin{tabular}{|l|l|l|l|l|l|}
\hline \multirow{2}{*}{ \%Dev } & \multirow{2}{*}{ Jitter } & \multicolumn{4}{|c|}{ MSE } \\
\cline { 3 - 6 } & & EDRBF & RBF & NN & IDW \\
\hline \multirow{4}{*}{10} & 0.0 & 0.6924 & 0.6926 & 0.7453 & 1.426 \\
\cline { 2 - 6 } & 0.1 & 0.7543 & 0.7551 & 0.8226 & 1.510 \\
\cline { 2 - 6 } & 0.2 & 0.8240 & 0.8230 & 0.9136 & 1.571 \\
\cline { 2 - 6 } & 0.3 & 1.0660 & 1.0658 & 1.2107 & 1.9240 \\
\hline \multirow{4}{*}{30} & 0.0 & 1.3501 & 1.3519 & 1.5814 & 2.3865 \\
\cline { 2 - 6 } & 0.1 & 1.2921 & 1.2936 & 1.5313 & 2.3127 \\
\cline { 2 - 6 } & 0.2 & 1.2909 & 1.2913 & 1.5254 & 2.2869 \\
\cline { 2 - 6 } & 0.3 & 1.3882 & 1.3879 & 1.6172 & 2.3623 \\
\hline \multirow{4}{*}{40} & 0.0 & 2.0254 & 2.0294 & 2.3637 & 3.0374 \\
\cline { 2 - 6 } & 0.1 & 2.1011 & 2.1052 & 2.4395 & 3.0930 \\
\cline { 2 - 6 } & 0.2 & 2.0009 & 2.0040 & 2.3131 & 2.9636 \\
\cline { 2 - 6 } & 0.3 & 2.1663 & 2.1682 & 2.5228 & 3.1392 \\
\cline { 2 - 6 } & 0.0 & 2.7634 & 2.7682 & 3.0312 & 3.4352 \\
\cline { 2 - 6 } & 0.1 & 2.7612 & 2.7653 & 3.0326 & 3.4361 \\
\cline { 2 - 6 } & 0.2 & 2.8715 & 2.8761 & 3.1139 & 3.4758 \\
\cline { 2 - 6 } & 0.3 & 3.1530 & 3.1580 & 3.0549 & 3.3699 \\
\hline
\end{tabular}




\section{CONCLUSIONS}

The super resolution algorithms based on RBF interpolation and EDRBF interpolation performed better than other popular algorithm as shown in the Table 1. The accuracy obtained by EDRBF is marginal better. It looks like EDRBF will perform much better for noisy images. Currently, we are testing and comparing EDRBF with other algorithms for noise conditions and registration errors. The results will be published in future confererences.

\section{REFERENCES}

[1] Chesley, B., Lutz, R, and Brodsky, R., Space Payload Design and Sizing (Chapter 9, pp. 255-272) in book by Wetz J.R and Larson, W.J., Space Mission Analysis and Design (Third Edition), published by Microcosm Press and Kluwer Academic Publishers, 1999.

[2] Yang, J., and Huang, T., Image super-resolution: Historical overview and future challenges, Google book library 2013.

[3] Nguyen, N., P. Milanfar, and G. Golub, "Efficient generalized cross-validation with applications to parametric image restoration and resolution enhancement”, IEEE Transactions on Image Processing, 10(9):1299-1308, September 2001.

[4] Eren, P. E., M.I. Sezan, and A. M. Tekalp. Robust, “Object-based high- resolution image reconstruction from lowresolution video", IEEE Transactions on Image Processing, 6(10):1446-1451, 1997.

[5] Irani, M and Peleg, S., “ CVGIP, Graphical Models and Image Processing,” Vol. 53, No.3, May 1991.

[6] R. C. Hardie, K. J. Barnard, and E. E. Armstrong. "Joint MAP registration and high resolution image estimation using a sequence of under sampled images," IEEE Transactions on Image Processing, 6(12):1621-1633, 1997.

[7] Lehmann, T. M., Gonner, C. and Spitzer, K., "Survey: Interpolation Methods in Medical Image Processing, IEEE Transactions on Medical Imaging," Vol. 18, No. 11, November 1999.

[8] Hou, H. S., and Andrews, H. C., “Cubic Splines for Image Interpolation and Digital Filtering,” IEEE Transactions on Acoustics, Speech, and Signal Processing, Vol. ASSP-26, No. 6, December 1978, pp.508-517

[9] Mareboyana, M., J. Le Moigne, and J. Bennett, "High resolution image reconstruction from projection of low resolution images differing in subpixel shifts ", Proc. SPIE 9870, Computational Imaging, 98700F (May 20, 2016)

[10]Wang, Q., W. Shi, W., and P. M. Atkinson, "Sub-pixel mapping of remote sensing images based on radial basis function interpolation,” ISPRS Journal of Photogrammetry and Remote Sensing, Volume 92, June 2014, Pages 1-15

[11] Wang, L., S. Xiang, G. Meng, H. Wu, and C. Pan, "Edge-Directed Single Image Super-Resolution Via Adaptive Gradient Magnitude Self -Interpolation", IEEE Trans on Circuits and Systems for Video Technology, Vol. 23, Issue 8, Aug 2013

[12] Xin Li, M. T. Orchard, "New edge-direct interpolation,” IEEE Transactions on Image Processing, Volume 10 Issue 10, October 2001 